\section{Bazyli Degórski}

Pontificia Università San Tommaso d'Aquino, Roma

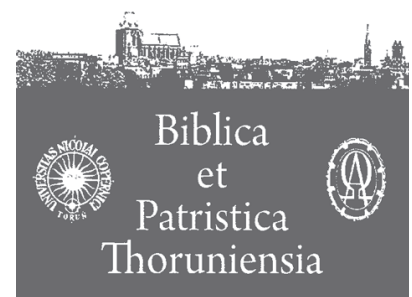

4 (2011) ISSN 1689-5150

\title{
La creazione dell'essere umano secondo la Prima Omelia di Origene al Libro della Genesi
}

\author{
The creation of the human being according \\ to the First Homily of Origen on the Book of Genesis
}

\section{Stworzenie człowieka w Pierwszej Homilii Orygenesa na Księgę Rodzaju}

Key words: Origen, creation, Book of Genesis, human being.

Słowa klucze: Orygenes, stworzenie, Księga Rodzaju, człowiek.

\section{Una breve introduzione}

T e Omelie di Origene sulla Genesi furono pronunciate dal Grande LAlessandrino a Cesarea in Palestina verso la metà del III secolo. Esse furono trascritte da tachigrafi mentre il predicatore ecclesiastico - Origene le predicava a Cesarea (all'epoca capitale ecclesiastica della Palestina, Patria del nostro Signore Gesù Cristo). Oggi noi possediamo questi frutti della predicazione origeniana nella traduzione di Rufino di Aquileia degli anni 400-404, traduzione discussa ma che, ciò nonostante, possiamo considerare fedele all'originale greco di Origene. In verità, la traduzione manoscritta che presenta le "Omelie sulla Genesi" del Grande Alessandrino non è affatto scarsa. Anzi, è addirittura numerosa': sedici omelie, mentre una diciassettesima "Omelia" pare sia sorta in Francia da una redazione utilizzata da sant'Isidoro di Siviglia².

1 Cf. M. I. Danieli (ed.), Origene, Omelie sulla Genesi [= "Collana di Testi Patristici", 14], Roma 1978, 27.

2 Cf. ibidem. 
Le Omelie di Origene, pronunciate a Cesarea in Palestina verso la metà del III secolo e frutto della predicazione di un Origene ormai maturo ed esperto, ci permettono di scorgere i particolari che rivelano l'Origene presbitero. La chiave interpretativa del Libro della Genesi, e anche di tutto l'Antico Testamento, è lo stesso Cristo Gesù. Infatti, il principio di tutti gli esseri creati è il Figlio di Dio, nel quale sono state create tutte le realtà esistenti e anche lo stesso essere umano, come esito fecondo della conversazione del Padre con il Figlio (cf. Gn 1, 26). Il Cristo è, dunque, la chiave interpretativa della Bibbia e ciò non solo a giudizio del Grande Alessandrino, ma anche di tutti gli altri Padri della Chiesa, senza alcuna differenza.

Per quanto riguarda la traduzione manoscritta delle opere di Origene, dobbiamo dire che i codici tramandati delle Omelie sulla Genesi sono numerosi. Larchetipo di essi è un testo composto in Campania nei secoli V e VI e trovato da Cassiodoro in un monastero vicino a Napoli, che egli riproduce per il suo Vivarium. Una copia del testo di Cassiodoro, che si conserva a Lione, risale ai secoli VI-VII ed è basilare per la formulazione del testo stesso ${ }^{3}$.

\section{Analisi dell'Omelia}

Nel presente articolo, analizziamo i capitoli dal dodicesimo sino alla fine della Prima Omelia di Origene al Libro della Genesi, ossia al capitolo diciasettesimo. Si tratta della sezione che analizza il pensiero del Grande Alessandrino in ordine alla creazione dell'essere umano.

In questi capitoli origeniani si incontra una tipica alternanza fra il significato "storico" degli eventi e quello spirituale, dal momento che il brano biblico viene interpretato dal Grande Alessandrino per allegoriam 4 . Il "dominio" dell'acqua

3 Cf. ibidem.

4 Cf. Origenes, Homilia Prima in Genesin 12, in: Origenes, Opera omnia quae graece vel latine tantum extant et eius nomine circumferuntur. Ex variis Editionibus et Codicibus manu exaratis, Gallicanis, Italicis, Germanicis et Anglicis collecta, recensita atque annotationibus illustrata, cum vita Auctoris et multis Dissertationibus ediderunt Carolus et Carol. Vincent. De la Rue Presbyteri et Monachi Benedictini e Congregatione S. Mauri. Denuo recensuit emendavit castigavit Carol Henric Eduard Lommatzsch, Tomus VIII, Berolini 1838, 119-120: "Quae qualiter intelligi per allegoriam debeant, exposuimus [...]” (“[...] abbiamo spiegato in qual modo queste cose si devono intendere allegoricamente [...]", in: M. I. Danieli (ed.), Origene, Omelie sulla Genesi [= "Collana di Testi Patristici", 14], Roma 1978, 51); Origenes, Homilia Prima in Genesin 15, in: Origenis, Opera omnia quae graece vel latine tantum extant et eius nomine circumferuntu, 126: "Videamus autem etiam per allegoriam quomodo ad imaginem Dei homo factus masculus et femina est" ("Ma vediamo 
(acque celesti) sulla terra è compreso come quello della mens, del sensus spiritalis che domina sulla materia, sulla "carne", cioè sul sensus carnis ${ }^{5}$.

Secondo ${ }^{6}$ quanto abbiamo spiegato sopra consegue che un uomo tale, quale lo abbiamo descritto, vuole esercitare il dominio sulle bestie già menzionate, ${ }^{7}$ sugli uccelli, rettili, quadrupedi, e su tutti gli altri; e abbiamo spiegato in qual modo queste cose si devono intendere allegoricamente, quando abbiamo detto che allacqua, cioè alla sua anima, si comanda di produrre sentimenti spirituali, e alla terra di far scaturire sentimenti carnali, affinché l’anima li domini, e non sia da essi dominata ${ }^{8}$.

A questo punto, viene espressa la dignità dell'essere uomo, che viene creato direttamente da Dio come le principali realtà cosmiche (il cielo e la terra, il sole e la luna), e la nozione di "immagine", come non riferibile al corpo ma all'"uomo

anche secondo l'allegoria come l'uomo sia stato fatto maschio e femmina, a immagine di Dio"), in: M. I. Danieli (ed.), Origene, Omelie..., 57); Origenes, Homilia Prima in Genesin 16, in: Origenis, Opera omnia quae graece vel latine tantum extant et eius nomine circumferuntu, 127: "Secundum allegoriam tamen in piscibus, et volatilibus, vel animalibus et repentibus terrae ea mihi videntur indicari, de quibus nihilominus superius diximus, id est, vel quae de sensu animae, et cordis cogitatione procedunt, vel quae ex desideriis corporalibus, et carnis motibus proferuntur [...]" ("Ma secondo l'allegoria, mi sembra che nei pesci, nei volatili, negli animali e rettili della terra, siano indicate le cose, delle quali non senza motivo abbiamo sopra parlato, cioè, o le cose che procedono dal sentire dell'anima e dalla riflessione del cuore, o quelle che scaturiscono dai desideri del corpo e dai movimenti della carne", in: M. I. Danieli (ed.), Origene, Omelie, 58-59); Origenes, Homilia Prima in Genesin 17, in: Origenis, Opera omnia quae graece vel latine tantum extant et eius nomine circumferuntu, 127: "Secundum allegoriam tamen herba terrae, et fructus eius, qui ad escam hominibus indulgetur, potest de affectibus intelligi corporalibus" ("Secondo l'allegoria, peraltro, nell'erba della terra e nei suoi frutti, accordati come cibo agli uomini, possono intendersi le passioni del corpo [...]", in: M. I. Danieli (ed.), Origene, Omelie, 59).

5 Cf. G. Sfameni Gasparro, Adamo, in: A. Monaci Castagno (ed.), Origene. Dizionario: la cultura, il pensiero, le opere, Roma 2000, 2.

6 Cf. ibidem 1, 11.

7 Cf. Origenes, Homiliae in Genesin 1, 8-11.

8 Ibidem 1, 12, in: Origenis, Opera omnia quae graece vel latine tantum extant et eius nomine circumferuntu, 119-120: "Et dixit Deus: faciamus hominem ad imaginem et similitudinem nostram, et principatum gerat piscium maris et volucrum coeli, et animalium et universae terrae, et omnium, quae repunt super terram". Consequenter secundum ea, quae superius exposuimus, talem hominem, qualem descripsimus, vult principatum gerere praedictarum bestiarum, et volucrum, et repentium, quadrupedum, et omnium reliquorum. Quae qualiter intelligi per allegoriam debeant, exposuimus, cum diximus iuberi aquam, id est, mentem eius spiritualem sensum producere, et terram sensum carnis proferre, ut dominetur iis mens, et non illa dominentur ei". 
interiore", in polemica con le diffuse concezioni antropomorfiche ${ }^{9}$. L'immagine secondo cui lessere umano è stato creato è Cristo-Logos (Xpıбтó $-\Lambda$ ójoc). Ciò nonostante, l'uomo creato "ad immagine di Dio", con il peccato, ha assunto l'immagine del diavolo che egli dovrà abbandonare acquistando la somiglianza di Cristo $^{10}$. La distinzione tra "immagine" in senso statico e "immagine" in senso dinamico, introdotta già da sant'Ireneo di Lione e da san Clemente di Alessandria, sollecita anche ad una proiezione futura dell' immagine stessa, che viene tematizzata maggiormente da Origene. Anchegli è attratto dal suggestivo e ricco concetto dell' $\in i \kappa \omega \nu$ di Dio, ma ne intravede subito il carattere venturo: la rivelazione perfetta dell'immagine, così come la sua realizzazione piena, appartengono al futuro, con le evidenti ripercussioni ascetiche e mistiche ${ }^{11}$.

\section{L'uomo immagine di Dio}

"Dunque guardiamo sempre questa immagine di Dio, per poter essere trasformati a sua somiglianza. Se infatti l'uomo, fatto a immagine di Dio, guardando - contro natura - l'immagine del diavolo, è diventato per il peccato simile a lui, molto di più, guardando l'immagine di Dio, a somiglianza della quale è stato fatto da Dio, mediante il Verbo e la potenza di lui, riceverà quella forma [di lui], che gli era stata data per natura. E nessuno, vedendo che la sua somiglianza è più col diavolo che con Dio, disperi di poter di nuovo ricuperare la forma dell'immagine di Dio, poiché il Salvatore non è venuto «a chiamare a penitenza i giusti, ma i peccatori» ${ }^{12}{ }^{\prime 3}$.

9 Cf. B. Degórski, Ojcowie Kościoła o "człowieku wewnętrznym" i o "człowieku zewnętrznym", "Ateneum Kapłańskie" 151 (2008) fasc. 1 (596) 105-118.

10 Cf. G. Sfameni Gasparro, Adamo, in: A. Monaci Castagno (ed.), Origene. Dizionario: la cultura, il pensiero, le opere, Roma 2000, 2.

11 Cf. B. Degórski, Visioni antropologiche dei Padri, in: B. Moriconi (ed.), Antropologia cristiana. Bibbia, teologia, cultura, Città Nuova, Roma 2001, 373-414 (passim).

12 Lc 5, 32; Mt 9, 13.

13 Origenes, Homilia Prima in Genesin 13, in: Origenis, Opera omnia quae graece vel latine tantum extant et eius nomine circumferuntu, op. cit., 124: "Semper ergo intueamur istam imaginem Dei, ut possimus ad eius similitudinem reformari. Si enim ad imaginem Dei factus homo, contra naturam intuens imaginem diaboli, per peccatum similis eius effectus est; multo magis intuens imaginem Dei, ad cuius similitudinem factus est a Deo, per Verbum et virtutem eius recipiet formam illam, quae data ei fuerat per naturam. Sed nemo desperet, videns similitudinem suam magis esse cum diabolo, quam cum Deo, posse se iterum recuperare formam imaginis Dei, quia non venit Salvator vocare iustos, sed peccatores in poenitentiam". 


\section{Maschio e femmina}

Alla creazione del "maschio e femmina" viene applicato il senso letterale, come anticipazione della futura creazione della donna, mentre secondo il senso spirituale essa indica l'uomo interiore formato ex spiritu et anima ${ }^{14}$.

«Maschio e femmina li fece, e li benedì Dio, dicendo: Crescete e moltiplicatevi, e riempite la terra e dominate su di essa» ${ }^{15}$. Sembra conveniente, in questo punto, ricercare secondo la lettera come, non essendo ancora stata fatta la donna, la Scrittura dica: "Li fece maschio e femmina". Forse, penso io, a motivo della benedizione con cui li ha benedetti, dicendo: "Crescete e moltiplicatevi, e riempite la terra", prevenendo quel che sarebbe accaduto, dice: "Maschio e femmina li fece", giacché in verità l'uomo non poteva crescere e moltiplicarsi, se non con la donna. Dunque, affinché si credesse che la sua benedizione senza dubbio si sarebbe attuata, dice: «Maschio e femmina li fece» ${ }^{16}$.

Qui Origene sviluppa quindi tutta la simbologia sponsale della coppia spirituale, dalla cui armonia deriva il dominio sulla "terra", ossia sul sensus carnis e sulle corporeae voluptates ${ }^{17}$.

Invero, se l'anima congiunta allo spirito e, per così dire, a lui coniugalmente unita ora si volge ai piaceri del corpo e piega il suo sentire ai godimenti carnali, ora sembra obbedire ai salutari ammonimenti dello spirito, ora cede ai vizi della carne: unanima siffatta cioè, come contaminata dall'adulterio col corpo, non si può dire che cresca e si moltiplichi legittimamente, poiché la Scrittura designa come imperfetti i figli degli adulteri ${ }^{18}$. Infatti, unanima simile che, lasciata da parte l'unione con lo spirito, si prostra tutta al sentire della carne e ai desideri del corpo, come

14 Cf. G. Sfameni Gasparro, Adamo, in A. Monaci Castagno (ed.), Origene. Dizionario: la cultura, il pensiero, le opere, Roma 2000, 2.

15 Gn 1, 27-28.

16 Origenes, Homilia Prima in Genesin 14, in: Origenis, Opera omnia quae graece vel latine tantum extant et eius nomine circumferuntu, 124-125: „Masculum et feminam fecit eos, et benedixit eos Deus, dicens: crescite et multiplicamini, et replete terram, et dominammi in ea». Dignum videtur hoc in loco requirere secundum literam, quomodo nondum facta muliere dicit Scriptura: «masculum et feminam fecit eos». Fortassis, ut ego arbitror, propter benedictionem, qua benedixit eos, dicens: "crescite et multiplicamini, et replete terram»: praeveniens, quod futurum erat, dicit: «masculum et feminam fecit eos»" (traduzione italiana: M.I. Danieli [ed.], Origene, Omelie, 56-57).

17 Cf. G. Sfameni Gasparro, Adamo, in: A. Monaci Castagno (ed.), Origene. Dizionario: la cultura, il pensiero, le opere, Roma 2000, 2.

18 Cf. Sap 3, 16. 
distoltasi da Dio spudoratamete, si sentirà dire: «La faccia ti è diventata faccia di meretrice, senza pudore ti sei resa per tutti» ${ }^{19}$. Dunque ${ }^{20}$, sarà punita come meretrice e si comanda che i suoi figli siano preparati per il massacro ${ }^{21}$.

Come abbiamo visto, il Grande Alessandrino nota che "il nostro uomo interiore consta di spirito e di anima: si dice maschio lo spirito, lanima si può denominare femmina"22. Egli indica nella concordia e nel consensus, con evidente riferimento al tema della $\sigma u \mu \phi \omega v i \alpha$ tra gli sposi (cf. 1 Cor 7, 5), i due elementi della condizione necessaria per la "generazione" dei figli, ossia di quei buoni pensieri capaci di popolare la "terra", identificata al subiectum sibi sensus carnis $^{23}$.

Loriginaria e originale definizione di essere umano, presente nelle pagine della Sacra Scrittura, dunque, implicò fin dall'inizio della riflessione cristiana la chiara consapevolezza della dignità che deriva all'essere umano dalla sua relazione a Dio, il suo carattere personale e la sua vocazione. Tale eccellenza, tuttavia, si trova immersa nella caducità della materia, composizione che rende l'essere umano una creatura singolare, perché impegnata in un dinamismo conflittuale - e libero - necessario per realizzare compiutamente le sue prerogative. Il corpo umano, tempio dell'anima dell'essere umano, non è necessariamente negativo, non è una prigione per l'anima; tuttavia, la maggioranza degli autori propende per collocare nel voûs, "la parte più sottile, superiore dell'anima", o nelle espressioni spirituali del soggetto umano, la sede della somiglianza; ciò non senza esasperazioni, soprattutto allorquando si amplifichi il discorso nella sua dimensione futura. Tale è, ad esempio, la posizione di Origene che,

19 Ger 3, 3.

20 Cf. Is $14,21$.

21 Origenes, Homilia Prima in Genesin 15, in: Origenis, Opera omnia quae graece vel latine tantum extant et eius nomine circumferuntu, 126: "Iam vero si anima coniuncta spiritui, atque eius, ut ita dicam, coniugio copulata, declinet aliquando ad corporeas voluptates, sensumque suum in delectationibus carnalibus inclinet, et aliquando quidem obtemperare videatur salutaribus monitis spiritus, aliquando vero vitiis carnalibus cedat, talis anima velut adulterio corporis maculata, neque crescere, neque multiplicari legitime dicitur, quoniam quidem filios adulterorum imperfectos Scriptura designat. Talis enim anima, quae spiritus coniunctione deserta, sensui se carnis, et desideriis corporalibus totam prosternit, velut aversa a Deo impudenter, audiet quia «facies meretricia facta est tibi, sine pudore effecta es ad omnes». Veluti meretrix ergo punietur, et filii eius ad occisionem praeparari iubentur" (traduzione italiana: M.I. Danieli (ed.), Origene, Omelie, 58).

22 Origenes, Homilia Prima in Genesin 15, in Origenis, Opera omnia quae graece vel latine tantum extant et eius nomine circumferuntu, 126: "Masculus spiritus dicitur, femina anima potest nuncupari” (traduzione italiana: M.I. Danieli (ed.), Origene, Omelie, 57).

23 Cf. ibidem. 
spingendo alle ultime conseguenze la tesi di una somiglianza noetica, in fase di elaborazione escatologica concepisce una reintegrazione a livello dello "spirito", o di "angelo" che, nonostante l'offuscamento dovuto alla tunica di pelle ${ }^{24}$, rimane sostanzialmente inalterato.

Questi spunti veterotestamentari reclamano un'interpretazione neotestamentaria, che fu elaborata dagli stessi autori in chiave cristologica, anche in questo caso non scevra da accentuazioni e tentennamenti, ma sostanzialmente coesa e suggestiva.

Origene è il primo a focalizzarne l'idea e la nomenclatura, all'occasione delle sue spiegazioni diffuse riguardo la creazione. L"uomo interiore" viene concepito come dicotomico rispetto all'uomo corporeo, materiale e segue una linea vitale opposta ${ }^{25}$. Per illustralo, possiamo citare questo brano origeniano che ottimamente mostra quanto diciamo:

All'inizio dei libri di Mosè, dove si descrive la creazione del mondo, viene narrata la creazione di due uomini, il primo fatto ad immagine e somiglianza di $\mathrm{Dio}^{26}$, il secondo plasmato dal fango della terra ${ }^{27}$. L'Apostolo Paolo, ben approfondito su questo argomento, ha scritto nelle sue lettere in maniera chiara e perspicua che in ognuno di noi ci sono due uomini. Infatti dice: Se il nostro uomo, che è di fuori, si corrompe, quello che è dentro si rinnova di giorno in giorno ${ }^{28}$; e ancora: Mi rallegro, infatti della legge di Dio secondo l'uomo interiore ${ }^{29}$; e scrive varie altre cose di questo genere. Ritengo perciò che nessuno debba dubitare che Mosè all'inizio della Genesi abbia descritto la creazione di due uomini: infatti vediamo Paolo, che certo comprendeva meglio di noi ciò che Mosè aveva scritto, parlare di due uomini che sono in ognuno di noi. Di questi uno, quello interiore, Paolo ci ricorda che si rinnova di giorno in giorno; l'altro invece, quello esteriore, nei santi e in quanti sono tali quali Paolo, si corrompe e si indebolisce ${ }^{30}$.

24 Origene pare abbia sempre oscillato tra cristianesimo e platonismo. Sul tema cf. M. Simonetti, Alcune osservazioni sull'interpretazione origeniana di Genesi 2, 7 e 3, 21, "Aevum" 36 (1962) 370-381.

25 Cf. B. Degórski, Visioni antropologiche dei Padri, in: B. Moriconi (ed.), Antropologia cristiana. Bibbia, teologia, cultura, Città Nuova, Roma 2001, 373-414 (passim).

26 Cf. Gn 1, 26.

27 Cf. Gn 2, 7.

282 Cor $4,16$.

$29 \operatorname{Rm} 7,22$.

30 Origenes, Commentarium in Canticum Canticorum, praefatio (traduzione italiana: M. Simonetti [ed.], Origene, Commento al Cantico dei Cantici [= Collana di testi patristici, 1], Roma 1976, 37-38), GCS 8, 63-67. 
Solo dell'uomo interiore si può predicare ed affermare l'essere immagine - o, meglio, l'essere ad immagine ( $\left.\kappa \alpha \tau^{\prime} \in i \kappa o ́ v \alpha\right)$ - in quanto si identifica con la parte superiore dellanima ( $\psi \cup \chi \eta َ)$, detta "intelletto" (voûs; mens, intellectus), che è la sede della ragione ( $\lambda$ ó $\gamma \circ \varsigma$ ) e, quindi, delle sue operazioni quali la conoscenza, il giudizio, la determinazione del bene morale. Solo questa dimensione eminente ed esclusiva dell'essere umano, rispetto alle altre creature, riflette l'influsso dello Spirito divino $(\Pi v \in \hat{v} \mu \alpha)$ :

Quando, dunque, nel progresso, si giunge al punto in cui si accoglie la Legge di Dio e si offrono voti al Signore - e non si possono offrire voti al Signore se non si ha in se stessi e nella propria sostanza di che offrire a Dio - l'uomo esteriore è incapace di accogliere la Legge di Dio e di offrire da solo dei voti, giacché non può avere qualcosa che sia degno di Dio, ma è piuttosto l'uomo interiore che ha in sé di che offrire a Dio: in lui, infatti, è la dimora delle virtù, in lui ogni intelligenza e scienza, in lui il rinnovarsi dellimmagine divina. Quando egli ha recuperato la propria immagine, nella quale dal principio è stato creato da $\mathrm{Dio}^{31}$, e che ha ricevuto, nel ristabilimento delle virtù, la bellezza della forma prima, allora può offrire voti a Dio e allora è detto non più soltanto uomo, ma uomo ${ }^{32}$ uomo ${ }^{33}$.

Origene ravvisa nell'insegnamento di san Paolo Apostolo, in modo inequivocabile, la dualità e, anzi, l’opposizione tra uomo esteriore e uomo interiore, che riflette il più ampio problema origeniano della teologia del visibile e dell'invisibile ${ }^{34}$, eco della sua posizione riguardo la natura della materia:

[...] le cose non corporali sono denominate con gli omonimi di tutte le cose corporali, e anche le cose corporali concernono l'uomo esteriore, e gli omonimi delle cose corporali - uomo interiore. La Scrittura dice che ci sono due uomini nelluomo: Se anche il nostro uomo esteriore si va disfacendo, quello interiore si rinnova di giorno in giorno ${ }^{35}$; e: Mi compiaccio nella legge di Dio secondo l'uomo interiore ${ }^{36}$. L'Apostolo presenta dappertutto questi due uomini distinguendoli. Perciò, a mio avviso, egli non è colui che di sua propria iniziativa inaugura questa dottrina, ma svincolandola

31 Cf. Mc 10, 6.

32 Cf. Nm 30, 3.

33 Origenes, In Numeros tractatus 24, 2 (traduzione italiana: M.I. Danieli [ed.], Origene, Omelie sui Numeri [= Collana di testi patristici, 76], Roma 1988, 332-333), GCS 7, 227-229.

34 Cf. O. Rousseau, Introduction, in: idem (ed.), Homélies sur le Cantique [SCh 37], Paris 1966, 21; A. Solignac, Homme intérieur. Age patristique, in: Dictionnaire de spiritualité, ascétique et mystique, doctrine et histoire, VII, Paris 1968, 654.

352 Cor $4,16$.

$36 \operatorname{Rm} 7,22$. 
dalle Scritture ove essa era espressa un po' confusamente, l'ha concepita ed espressa in un modo più spoglio ${ }^{37}$.

\section{Facciamo l'uomo secondo la nostra immagine e la nostra somiglianza}

Acclamata l'esistenza dell'uomo interiore, Origene procede a delinearne i tratti e le prerogative, in analogia con la struttura dell'uomo esteriore, attraverso una descrizione suggestiva ed esauriente che costituisce un esempio notevole di interpretazione allegorica della Scrittura e che vale essere riportata integralmente ${ }^{38}$ :

Così allora, al tempo della creazione dell'uomo, fu prima creato l'uomo secondo l'immagine, presso il quale non si trova la materia, perché l'uomo secondo l'immagine non è stato fatto dalla materia: E Dio ha detto: «Facciamo l'uomo secondo la nostra immagine e la nostra somiglianza e che comandi, ecc.» ${ }^{39}$ E Dio creò l'uomo non prendendo una zolla di terra ${ }^{40}$, ma egli lo creè a immagine di Dio ${ }^{41}$. [...]. Ci sono, quindi, due uomini in ciascuno di noi. [...]. Si può dire che ogni membro dell'uomo esteriore si ritrova, sotto questo nome, nell'uomo interiore. L'uomo esteriore ha due occhi. Anche dell'uomo interiore si dice che ha due occhi: Illumina i miei occhi, affinché io non mi addormenti mai nella morte $e^{42}$. Non si tratta qui degli occhi, né del sonno sensibile, né della morte nel senso corrente. Il precetto del Signore luminoso rischiara i nostri occh ${ }^{43}$. Non è che osservando i precetti del Signore acquistiamo una vista più penetrante per quanto riguarda il corpo, ma osservando i precetti divini acquistiamo una vista più penetrante riguardo la ragione. Gli occhi dell'uomo interiore sono più perspicaci che noi stessi. Togli il velo dai miei occhi e scruterò le meraviglie della tua legge $e^{4}$. Questo vuol dire che i suoi occhi erano velati? Affatto, ma «i nostri occhi» [sta] nel senso della nostra ragione. Solo Gesù può togliere questo velo, affinché possiamo contemplare ciò che egli ha scritto e capire pienamente ciò che ha detto in termini velati. L'uomo esteriore ha gli orecchi. Anche

\footnotetext{
37 Origenes, Disputa cum Heraclide 11, SCh 67, 78-80.

38 Il testo è tradotto da chi scrive, non esistendo ancora - a quanto pare - una traduzione ufficiale in lingua italiana.

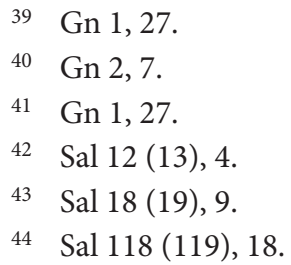


dell'uomo interiore è detto che ha gli orecchi. Chi ha orecchi da intendere, intenda ${ }^{45}$. Tutti hanno questi orecchi che sono organi dei sensi, ma non tutti riescono a custodire puri i loro orecchi interiori. I primi non dipendono da noi, i secondi dipendono. Proprio perché dipende da noi il possedere questi orecchi interiori, il profeta dice: Ascoltate, o sordi! O ciechi, alzate gli occhi per vedere! E chi è sordo se non i miei servitori? E chi è cieco se non i loro maestri? E gli schiavi di Dio sono diventati ciech $^{46}$. Che dipenda da noi diventare sordi. [...] per quanto riguarda questi orecchi interiori, ascoltatelo dalla bocca del profeta: I peccatori si sono smarriti dalla loro nascita. Hanno errato subito dal seno di loro madre. Hanno detto menzogne. Il furore è in essi, simile a quello del serpente, come quello di un aspide sordo che si tura gli orecchi, che non ascolterà la voce degli incantatori e l'incantesimo che gli rivolge un uomo esperto ${ }^{47}$. Anche voi tutti che avete consapevolezza di non essere senza macchia, se ascoltate la parola e l'incantesimo che gli rivolge un uomo esperto ${ }^{48}$, se ascoltate le parole che cercano di incantarvi per reprimere il vostro furore e la vostra meschinità, e in tal modo vi turate gli orecchi anziché aprirli del tutto per accogliere queste parole, a voi si applicheranno le parole: Il furore è in essi, simile a quello del serpente, come quello di un aspide sordo che si tura gli orecchi, che non ascolterà la voce degli incantatori e l'incantesimo che gli rivolge un uomo esperto ${ }^{49}$. L'uomo esteriore ha le narici per sentire, e percepisce il buono e il cattivo odore. E l'uomo interiore, per percepire il buon odore della giustizia e il cattivo odore dei peccati, ha altre narici. Del buon odore ammonisce l'Apostolo quando dice: In ogni luogo noi siamo per Dio il buon odore di Cristo tra quelli che si salvano e tra quelli che si perdono: per gli uni odore di morte per la loro morte, per gli altri odore di vita per la loro vita ${ }^{50}$. E anche Salomone dice nel «Cantico dei Cantici» per la bocca delle giovani donne, figlie di Gerusalemme: Dietro a te correremo allodore dei tuoi profumi $^{51}$. Perciò, come attraverso le narici percepiamo il buon odore sensibile e il cattivo odore sensibile, così esiste nell'ordine dell'uomo interiore, presso colui che è sano quanto agli organi sensoriali divini, una percezione: del buon odore della giustizia, quella che aveva l'Apostolo, e il cattivo odore dei peccati. Qual è il cattivo odore dei peccati? Quello di cui parla il profeta in questi termini: Le mie piaghe sono divenute fetore e putrefazione a causa della mia follia ${ }^{52}$. L'uomo esteriore è dotato del gusto. L'uomo interiore anche è dotato del gusto spirituale, a proposito del quale è detto: Gustate e vedete come dolce è il Signore ${ }^{53}$. L'uomo esteriore possiede un tatto

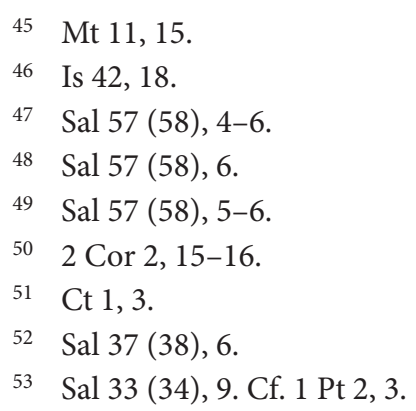


di ordine sensibile. L'uomo interiore anche possiede un tatto. È quel tatto attraverso il quale l'emorroissa toccò il lembo del mantello di Gesù. Lo toccò; ne fu resa testimonianza per colui che dice: Chi mi ha toccato?54. Ora, un attimo dopo, Pietro gli disse: La folla ti preme e tu domandi: chi mi ha toccato? ${ }^{55}$. Nel suo modo di pensare, quando si tocca, ciò avviene per un contatto corporale, non spirituale. Eppure coloro che premevano Gesù non lo toccavano, perché essi non lo toccavano con la fede. Soltanto questa donna, possedendo un certo tatto divino, toccò Gesù e per questo fu guarita. E poiché ella lo toccò con un tatto divino, a causa di ciò una forza uscì da Gesù per rispondere a questo tatto divino. Egli anche dice: Chi mi ha toccato? Perché ho sentito che la forza è uscita da $m e^{56}$. Proprio a proposito di questo tatto divino dice Giovanni: E ciò che le nostre mani hanno toccato, ossia il Verbo di vita ${ }^{57}$. Così allora noi possediamo delle altre mani e proprio di esse è detto: Che l'elevazione delle mie mani sia un sacrificio della sera ${ }^{58}$. Perché se elevo queste mani, ma lascio pendere le mani della mia anima, anziché levarle per le opere sante e buone, non è vero che l'elevazione delle mie mani sia un sacrificio della sera. Ho ugualmente altri piedi ai quali si rapporta il precetto che mi dà Salomone quando dice: Il tuo piede non inciamperà ${ }^{59}$. Si legge una strana cosa nell' «Ecclesiaste». Senza dubbio, a chi non la comprende essa sembrerà insensata, ma l'Ecclesiaste l'ha detta proprio per il saggio: Il saggio ha $i$ suoi occhi nella sua testa ${ }^{60}$. In quale testa? Ogni uomo, anche l'insensato e il pazzo, ha i propri occhi corporali nella testa corporale. Però, il saggio ha i suoi occhi - quelli di cui già abbiamo parlato, quelli che sono illuminati dal precetto del Signore - nella sua testa, nel Cristo, perché l'Apostolo dice che Cristo è la testa dell'uomo ${ }^{61}$. Il principio pensante è nel Cristo. Le mie viscere, le mie viscere sono doloranti62 ${ }^{6}$, dice Geremia. Quali viscere gli dolgono? Quelle che anche a noi sono doloranti, quelle che, finché esse soffrono per il nostro popolo i dolori del parto, fanno sì che le mie viscere siano doloranti, e i miei sensi, non questi [carnali], ma quelli del mio cuore. Se passo alle parti fini del corpo, le percepisco, sotto forma non carnale, nell'anima. Signore, non mi riprendere nel tuo furore, e non mi castigare nella tua ira. Abbi pietà di me, Signore, perché sono debole. Guariscimi, Signore, perché le mie ossa sono sconquassate ${ }^{63}$. Quale osso del profeta era sconquassato? La costituzione della sua anima e la fermezza della sua ragione erano sconquassate, ed egli implora la guarigione proprio di questo osso. Le nostre ossa

Lc 8, 45 .

Ibidem.

Lc 8, 46.

$1 \mathrm{Gv} 1,1$.

Sal 140 (141), 2.

Pro 3, 23.

Qo 2, 14.

1 Cor 11, 3.

Ger 4, 19.

Sal 6, 2-3. 
sono state disperse negli inferi ${ }^{64}$. Considera - te ne prego - il peccatore, considera la sua condizione di permanere nel peccato, di permanere nella morte, di soggiornare nel male. Dirai di un tale uomo che le sue ossa sono state disperse. Tutte le mie ossa diranno: Signore, chi è pari a te ${ }^{65}$ Ecco le ossa che parlano, conversano, si esprimono, sentono Dio e, malgrado ciò, quell'insensibilità di cui testimoniano i medici, che segano le ossa del paziente senza che egli senta niente sotto l'azione della sega. Quindi, tutte le mie ossa diranno: Signore, chi è pari a te? Tutte queste ossa sono l'uomo interiore che le possiede. L'uomo interiore ha un cuore. Uditemi, voi che avete perduto il vostro cuore ${ }^{66}$. Essi possedevano un cuore, quello del corpo, perché non si perde mai quel cuore. Quando, però, un uomo ha smesso di coltivare il suo essere razionale e, a causa del non agire, il principio della ragione è sparito in lui, egli ha perso il suo cuore e a tale uomo si dice: Uditemi, voi che avete perduto il vostro cuore. Anche i capelli della vostra testa sono tutti contati ${ }^{67}$. Quali capelli? Quelli per i quali essi erano spiritualmente nazirei. Poiché ritrovi tutti gli elementi del corpo materiale nell'uomo interiore, non dubitare più che anche il sangue, sotto lo stesso nome del sangue materiale, e tutto come le altre parti del corpo, esistono nell'uomo interiore ${ }^{68}$.

Lo sdoppiamento tra uomo interiore ed esteriore, e la loro analogia, viene esteso anche alle funzioni umane, per cui si hanno due intelligenze ( $\gamma \psi \cup \chi \eta$ ' $\Omega$ voûs), due amori ("̌ $\rho \omega \varsigma$ ' $\Omega \dot{\alpha} \gamma \alpha \dot{\alpha} \pi \eta)$, e due compiti morali, giacché l'uomo interiore è tenuto a diventare un perfetto Israelita - secondo l'etimologia della parola "Israele" che significa "capace di vedere Dio" -, vocazione che Origene ravvisa tra le pieghe della rivelazione: "Usci dunque il figlio di una donna israelita, ed era figlio di un egiziano fra i figli d'Israele; e litigarono nel campo il figlio della donna israelita e un uomo israelita ${ }^{69}$. Vedi quale precauzione cè nella Scrittura divina: se sei attento, se la esamini con cura: colui che è nato da madre israelita e da padre egiziano non lo ha detto uomo, mentre quello che era israelita per l'una e l'altra razza, lo ha chiamato uomo. Pensiamo che queste cose siano scritte a caso? Pensiamo casuale che uno non sia detto uomo e l'altro lo sia? Non sono cose fortuite, vi è una ragione. Niente è scritto nelle parole di Dio senza una ragione profonda. Colui che era per una parte egiziano e per una parte israelita non meritava ancora di essere chiamato uomo; ma colui che era del tutto israelita, cioè che vedeva Dio con lo spirito, costui è chiamato

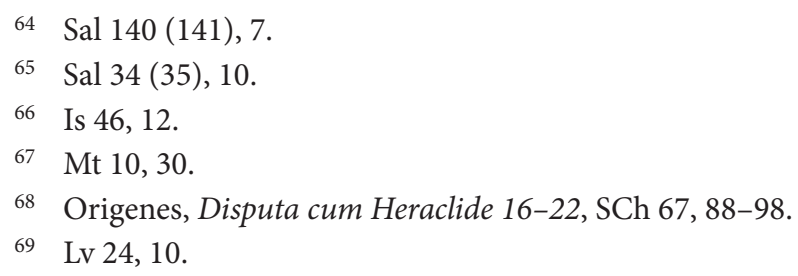


uomo: quell'uomo interiore che è stato fatto a immagine $d i D_{i o}^{70}$ e può vedere $e^{71}$ Dio"72.

All'uomo peccatore spetta, dunque, ritrovare l'uomo interiore ${ }^{73}$, a imitazione della donna del Vangelo ${ }^{74}$ che ha spazzato la sua casa per trovare la dramma perduta:

Poiché è presente il Verbo di Dio, e questa è ora la sua operazione: rimuovere la terra dall'anima di ognuno di voi, e aprire la tua fonte. Infatti è in te, e non viene dal di fuori, così come è in te il regno di $\mathrm{Dio}^{75}$. La donna che aveva perduto la dracma, non la trovò al di fuori, ma nella sua casa, dopo che ebbe accesa la lucerna e spazzata la casa $^{76}$ dalle sporcizie e dalle immondezze che per lungo tempo vi avevano accumulato la pigrizia e la stupidità, e là trovò la dracma. Anche tu, se accenderai la lucerna, se ricorrerai alla illuminazione dello Spirito Santo, e nella sua luce vedrai la luce $e^{77}$, troverai in te la dracma: giacché in te è stata posta l'immagine del re celeste. Quando Dio, da principio, fece l'uomo, lo fece a sua immagine e a sua somiglianza ${ }^{78}$, e pose questa immagine non allesterno, ma dentro di lui. Ma essa non poteva essere vista in te, fino a che la tua casa era sporca per le immondizie, e piena di ruderi. Questa fonte della scienza si trovava in te, ma non poteva fluire, poiché i Filistei l'avevano riempita di terra, e avevano fatto in te l'immagine del terrestre ${ }^{79}$. Ma se tu hai portato allora l'immagine del terrestre, ora, dopo avere ascoltato queste parole, purificato mediante il Verbo di Dio da tutto quel peso e oppressione, fa' risplendere in te l'immagine del celeste. Questa è l'immagine della quale il Padre diceva al Figlio: Facciamo gli uomini ad immagine e somiglianza nostra ${ }^{80}$. Il Figlio di Dio è il pittore di questa immagine: e poiché tale e così grande è il pittore, la sua immagine può essere oscurata dall'incuria, ma non può essere cancellata per la malvagità.

\section{Cf. Gn 1, 27.}

71 Cf. Mt 5, 8.

72 Origenes, In Leviticum tractatus 14, 3 (traduzione italiana: M.I. Danieli [ed.], Origene, Omelie sul Levitico [= Collana di testi patristici, 51], Roma 1985, 289), GCS 6/1, 484.

73 Cf. Origenes, In Epistulam ad Romanos tractatus 7, 4.

74 Cf. Lc 15, 8.

75 Cf. Lc 17, 21.

76 Lc 15, 8 .

77 Sal 36 (35), 10.

78 Cf. Gn 1, 26; Gn 5, 1.

79 Cf. 1 Cor 15, 49.

80 Gn 1, 26. 
Infatti l'immagine di Dio rimane sempre, anche se tu vi sovrapponi l'immagine del terrestre ${ }^{81}$.

La dottrina dell'uomo interiore, in Origene, dunque, rispecchia l'azione totale della Trinità: del Padre che imprime l'immagine, del Figlio che rimuove la terra con la sua azione redentrice, e dello Spirito che illumina e orienta l'agire umano. L'espressione e il concetto di "uomo interiore" è presente anche nell'ascetica di san Giovanni Cassiano, rappresentante della tradizione spirituale che si appella ad Evagrio Pontico ${ }^{82}$ e che suscitò ampi consensi non solo in Siria, ma anche nel mondo bizantino e - proprio attraverso san Giovanni Cassiano - in Occidente ${ }^{83}$.

Nel pensiero di Origene si scorgono toni più pesanti circa la necessità della liberazione dalla materia ai fini di un recupero dello stato primordiale di intelligenza, voûs, non decaduta ${ }^{84}$. Nella sua impostazione, evidentemente, riecheggia la teoria della duplice creazione dell'uomo, secondo la quale solo l'uomo interiore è invisibile, incorporeo, incorrotto, immortale ${ }^{85}$ e predisposto a contenere l'immagine divina ${ }^{86}$; il corpo, in quanto conseguenza del peccato, è escluso dal ritorno a Dio. La nostra principale sostanza ci è stata data in quanto siamo stati formati a immagine del Creatore; non si tratta però di quella che ci

81 Origenes, In Genesin tractatus 13, 4 (traduzione italiana: M.I. Danieli [ed.], Origene, Omelie sulla Genesi [= Collana di testi patristici, 14], Roma 1978, 206-207), GCS 8, 119. Origene spiega nello stesso modo l'espressione "uomo interiore" anche altrove. Cf. ad esempio: Origenes, In Genesin tractatus 1, 13. 15, GCS 6/1, 15 e 19; Origenes, In Exodum tractatus 2, 2, GCS 6/1, 157; ibidem, 8, 6, GCS 6/1, 230; ibidem, 9, 4, GCS 6/1, 243; ibidem, 10, 3, GCS 6/1, 248; Origenes, In Leviticum tractatus 1, 1, GCS 6/1, 280; ibidem, 3, 7, GCS 6/1, 312; Origenes, In Hieremiam tractatus 1, 13, GCS 3, 10-11; Origenes, Commentarius in Epistulam ad Romanos 1, 19, PG 14, 871 A; ibidem, 2, 13, PG 14, 912D-913; Origenes, De principiis 4, 3, 7, GCS 5, 333; ibidem, 4, 4, 9, GCS 5, 363; Origenes, Contra Celsum 2, 48, GCS 1, 170; ibidem, 6, 63, GCS 2, 134; ibidem, 7, 38, GCS 2, 188; ibidem, 7, 46, GCS 2, 197. Per tale visione antropologica, cf. anche, tra gli altri studi: H. Rahner, Das Menschenbild des Origenes, "Eranos-Jahrbuch" 15 (1947) 197-248; H. Crouzel, Théologie de l'image de Dieu chez Origène, Paris 1956, 150-157.

82 A proposito di Evagrio Pontico, cf. ad esempio: J. Gribomont, Evagrio Pontico, in: A. Di Berardino (ed.), Dizionario patristico e di antichichità cristiane, I, Casale Monferrato 1983, 1313-1314; J. Discroll, The "Ad Monachos" of Evagrius Ponticus [= Studia Anselmiana, 104], Roma 1991.

83 Cf. B. Studer, Una teologia monastica, in: A. Di Berardino, B. Studer (ed.), Storia della teologia. I: Epoca patristica, Casale Monferrato 1993, 619.

84 Cf. Origenes, De principiis 3, 6, 1.

85 Cf. idem, In Genesin homilia 1, 13.

86 Cf. idem, In Romanos 1, 19. 
viene dalla caduta per il corpo che abbiamo ricevuto, plasmato dal fango della terra ${ }^{87}$.

Lo schema usato induce Origene a modulare tutto il suo discorso sulla simbologia nuziale e sul carattere femminile dell'anima, collocata in posizione di netta inferiorità rispetto allo spiritus. Infatti, su di essa incombe la responsabilità di un'eventuale declinazione verso le passioni terrene e corporee, presentata come un adulterio, per cui sarà punita come meretrice. L'applicazione della differenziazione maschio-femmina alla struttura interiore dell'uomo permette un'esegesi del testo biblico coerente con il presupposto, essenziale nell'antropologia origeniana, della pertinenza della qualità di "immagine" alla componente spirituale. Resta, nello stesso tempo, aperto il problema della relazione tra quella qualità e le distinzioni sessuali peculiari della sfera corporea, materiale ${ }^{88}$.

\section{I santi e i peccatori}

Dopo un richiamo allesegesi letterale del "dominio" esercitato sugli animali da parte della prima coppia, è proposta quella del senso spirituale, quale dominio sui vizi, sia che essi scaturiscano dall'anima o dal corpo ${ }^{89}$.

«E dominate sui pesci del mare, e sui volatili del cielo, e sui giumenti, e su tutti [gli animali] che sono sopra la terra, e sui rettili che strisciano sopra la terra» ${ }^{90}$. Abbiamo già interpretato queste cose secondo la lettera, avendo detto che Dio disse: «Facciamo l'uomo», e il resto, quando dice: «E domini sui pesci del mare e sui volatili del cielo»"11, e le altre cose. Ma secondo l'allegoria, mi sembra che nei pesci, nei volatili, negli animali e rettili della terra, siano indicate le cose, delle quali non senza motivo abbiamo sopra parlato, cioè, o le cose che procedono dal sentire dellanima e dalla riflessione del cuore, o quelle che scaturiscono dai desideri del corpo e dai movimenti della carne. Invero i santi, conservando in sé stessi la benedizione di Dio, dominano queste cose, muovendo tutto l'uomo secondo

87 Cf. idem, In Iohannem 20, 22.

88 Cf. G. Sfameni Gasparro, Anima, in: A. Monaci Castagno (ed.), Origene. Dizionario: la cultura, il pensiero, le opere, Roma 2000, 19.
89 Cf. ibidem, 2.
90 Gn 1, 28.
91 Gn 1, 26. 
il volere dello spirito; invece i peccatori sono piuttosto dominati da queste stesse cose, che scaturiscono dai vizi della carne e dai piaceri del corpo ${ }^{92}$.

\section{Le passioni del corpo}

Anche questo testo origeniano ci fa vedere l'intreccio tra il piano storico e quello simbolico ${ }^{93}$, e tale modo esegetico pervaderà tutto il testo dell'Omelia dell'Alessandrino. Infatti, commentando Gn 1, 29-3094, Origene scruta, dapprima, il suo significato letterale e nota che, agli stessi primordi della storia dell'uomo, Dio permise agli esseri umani che si usassero come cibo erbe, ossia legumi e frutti degli alberi. Ciò nonostante, dopo il diluvio, Dio permise agli uomini di cibarsi anche di carni ${ }^{95}$. A questo punto, Origene interpreta il brano scritturistico "secondo l'allegoria" 96 , per la quale l'erba e i frutti della terra, dati in cibo agli uomini, possono essere intesi come le passioni del corpo, ad esempio: "l'ira e la concupiscenza sono germi corporali; i frutti,

92 Origenes, Homilia Prima in Genesin 16, in: Origenis, Opera omnia quae graece vel latine tantum extant et eius nomine circumferuntu, 126-127: „«Et dominamini piscium maris, et volatilium coeli, et iumentorum, et omnium, quae sunt super terram, et repentium, quae repunt super terram». Iam haec interpretata sunt secundum literam, cum diceremus, quod dicit Deus: «faciamus hominem», et reliqua, ubi dicit: «et dominetur piscium maris, et volatilium coeli», et cetera. Secundum allegoriam tamen in piscibus, et volatilibus, vel animalibus et repentibus terrae ea mihi videntur indicari, de quibus nihilominus superius diximus, id est, vel quae de sensu animae, et cordis cogitatione procedunt, vel quae ex desideriis corporalibus, et carnis motibus proferuntur: quorum sancti quique et benedictionem Dei in semet ipsis servantes, dominationem exercent, agentes totum hominem secundum spiritus voluntatem. Peccatoribus vero magis ipsa dominantur, quae de carnis vitiis, et corporis voluptatibus proferuntur" (traduzione italiana: M.I. Danieli [ed.], Origene, Omelie, 58-59).

93 Cf. G. Sfameni Gasparro, Adamo, in: A. Monaci Castagno (ed.), Origene. Dizionario: la cultura, il pensiero, le opere, Roma 2000, 2.

94 Cf. Origenes, Homilia Prima in Genesin 17, in: Origenis, Opera omnia quae graece vel latine tantum extant et eius nomine circumferuntu, 127: "Et dixit Deus: ecce dedi vobis omne foenum seminale, quod seminat semen, quod est super omnem terram, et omne lignum, quod habet in se fructum seminis seminalis, vobis erit ad escam, et omnibus bestiis terrae, et omnibus volatilibus coeli, et omnibus repentibus, quae repunt super terram, quae habent in se animam vitae".

95 Cf. Gn 9, 3 s. Cf. Origenes, Homilia Prima in Genesin 17.

96 Origenes, Homilia Prima in Genesin 17, in: Origenis, Opera omnia quae graece vel latine tantum extant et eius nomine circumferuntu, 127: "Secundum allegoriam [...]"(traduzione italiana: M.I. Danieli [ed.], Origene, Omelie, 59). 
ossia le opere, di tali germi, sono comuni a noi, esseri razionali, e alle bestie della terra" ${ }^{\prime 2}$.

Quando ci adiriamo per la giustizia, spiega il Grande Alessandrino, e ci nutriamo di questo frutto della terra, l'ira del corpo diviene nostro cibo ${ }^{98}$. Per confermarlo, l'Alessandrino si appella

al libro dei Numeri, e ricorda quel che fece il sacerdote Finees, il quale, avendo veduto una mertrice della gente di Madian aderire con amplesso impuro a un uomo di Israele, sotto gli occhi di tutti, riempito dall'ira dello zelo divino, afferrata una spada, li trapassò entrambi nel petto ${ }^{99}$; questo atto gli fu computato da Dio a giustizia, poiché il Signore dice: «Finees ha placato il mio furore, e gli sarà computato $^{100}$ a giustizia» ${ }^{101}$.

In tal modo, detto cibo materiale dell'ira diventa anche nutrimento dell'essere umano, quando egli ne usa spiritualmente per ottenere la giustizia. Se, però, egli causa l'ira in un modo non spirituale, tale suo comportamento formerà il cibo delle bestie, degli animali, perché di questi cibi si nutrono anche i demoni, che si cibano delle nostre cattive azioni e le fomentano ${ }^{102}$.

Il Grande Alessandrino, quindi, per confermare il suo pensiero, si appella

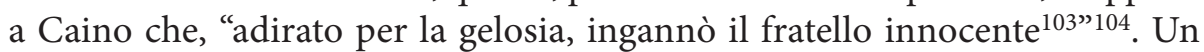
simile ragionamento deve essere applicato alla concupiscenza e a tutte le altre

97 Origenes, Homilia Prima in Genesin 17, in: Origenis, Opera omnia quae graece vel latine tantum extant et eius nomine circumferuntu, 127: "[...] ira et concupiscentia germen est corporis. Huius germinis fructus, id est, opus, nobis rationalibus, et bestiis terrae communis est" (traduzione italiana: M.I. Danieli [ed.], Origene, Omelie, 59).

98 Cf. Origenes, Homilia Prima in Genesin 17.

99 Cf. Nm 25, 7-8.

100 Cf. Nm 25, 11-13; Sal 106 (Volgata 105), 30-31.

101 Origenes, Homilia Prima in Genesin 17, in: Origenis, Opera omnia quae graece vel latine tantum extant et eius nomine circumferuntu, 128: “[...] ad librum Numerorum, et recordare, quid egerit Phinees sacerdos, qui cum Madianiticae gentis meretricem cum Israelita viro sub oculis omnium impuris vidisset inhaerere complexibus, ira divinae aemulationis impletus; arreptum gladium per utriusque pectus exegit. Quod opus reputatum est ei a Deo ad iustitiam, dicente Domino: «Phinees sedavit furorem meum, et reputabitur ei ad iustitiam»" (traduzione italiana: M.I. Danieli [ed.], Origene, Omelie, 60).

102 Cf. Origenes, Homilia Prima in Genesin 17.

103 Cf. Gn 4, 8.

104 Origenes, Homilia Prima in Genesin 17, in: Origenis, Opera omnia quae graece vel latine tantum extant et eius nomine circumferuntu, 128: “[...] Cain, qui innocentem fratrem ira decepit invidiae” (traduzione italiana: M.I. Danieli (ed.), Origene, Omelie, 60). 
passioni negative dell'essere umano ${ }^{105}$. Infatti, la concupiscenza può essere un moto positivo o un moto negativo, a seconda del traguardo, della meta della sua brama: quando essa desidera il Dio vivente ${ }^{106}$, la concupiscenza è positiva; ma quando essa si indirizzata a qualche realtà contraria alla legge divina ${ }^{107}$, la concupiscenza diventa cibo bestiale, animale. Per affermarlo, il Grande Alessandrino induce la vicenda di Acab, di Gezabele e di Nabot di Iezreel $^{108}$.

Lo stesso concetto viene subito, e ancora una volta, ribadito da Origene che loda la prudenza della Bibbia la quale, "avendo detto, riguardo agli uomini, che Dio disse: «Ecco vi ho dato ogni seme seminale, che è sopra la terra, e ogni albero, che è sopra la terra; vi sarà di cibo» ${ }^{109}$; riguardo alle bestie non ha detto: Diedi loro tutte queste cose in cibo, ma ${ }^{110}$ : «Sarà loro di cibo»"111. Etutto ciò, secondo il senso spirituale esposto da Origene, significa che queste passioni sono state date da Dio all'uomo, e che tuttavia saranno anche cibo per le bestie ${ }^{112}$.

Il Grande Alessandrino ribadisce, per concludere il suo pensiero, che la Sacra Scrittura prudentemente afferma che Iddio dice agli uomini: "Vi ho dato queste cose in cibo" ma, quando passa alle bestie, dice che queste cose saranno di cibo anche per gli animali ${ }^{113}$.

Tutta la Prima Omelia di Origene al libro della Genesi termina appellandosi al Nuovo Testamento o, meglio, a testi cristiani e, in specifico, a quelli di san Paolo Apostolo: "«il sentimento di Cristo» ${ }^{114}$, «le cose che ci sono state donate da Dio» ${ }^{15}$ e, delle cose che sono state date a noi per cibo, non facciamo

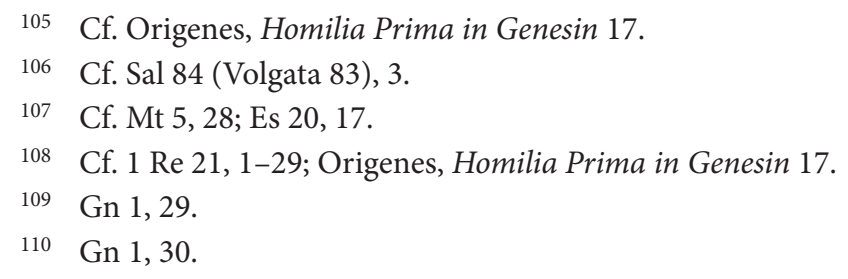

111 Origenes, Homilia Prima in Genesin 17, in: Origenis, Opera omnia quae graece vel latine tantum extant et eius nomine circumferuntu, 129: "[...] Observanda sane est sanctae scripturae etiam in verborum ratione cautela, quae, cum de hominibus dixisset, quia dixit Deus: «ecce dedi vobis omne semen seminale, quod est super terram, et omne lignum, quod est super terram, vobis erit ad escam»: de bestiis non dixit: quia dedi is ad escam haec omnia: sed: «erit illis ad escam» [...]” (traduzione italiana: M.I. Danieli (ed.), Origene, Omelie, 61).
112 Cf. Origenes, Homilia Prima in Genesin 17.
113 Cf. ibidem 17.
1141 Cor 2, 16.
1151 Cor 2, 12. 
cibo di porci o di cani ${ }^{116^{\prime \prime 117}}$. Tali testi, alla luce dei quali va letto il brano veterotestamentario in questione, devono renderci "degni di ricevere, nella dimora del nostro cuore, il Verbo e Figlio di Dio che viene con il Padre suo e vuole abitare presso di noi nello Spirito Santo ${ }^{118}$, del quale prima dobbiamo essere tempio per la santità ${ }^{119 " 120}$.

\section{Summary}

The present essay analyzes the First Homily of Origen on the Book of Genesis, from the $12^{\text {th }}$ chapter to the end of the Homily in the $17^{\text {th }}$ chapter, thus covering the section concerning the doctrine of the Great Alexandrine about the creation of the human being.

The chapters present a typical feature of the origenian way of proceeding in exegesis: the alternation between the "historical" and the "spiritual" meaning of the events narrated, since he interprets the Biblical passages per allegoriam. The "dominion" over the waters (the heavenly waters) on earth is understood as that of the mens, the sensus spiritalis ruling over matter, over the "flesh", namely over the sensus carnis.

The dignity of the human being is widely stated since the human being is directly created by God, just as the fundamental cosmic realities (the heavens, the earth, the sun and the moon), and the notion of "image" shall be understood as referring not to the body, but rather to the "interior human being", in clear opposititon to the widespread anthropological conceptions of his times. The image according to which the human being is created is the Christ-Logos; nevertheless, the human being created "at the image of God" has gained, through sin, the image of the devil, which he is called to abandon in order to assume the likeness of Christ.

The literal sense is applied to the creation of the "male and the female" inasmuch as anticipation of the future creation of the woman, whereas according to the spiritual sense such creation indicates the interior human being, formed ex spiritu et anima. Origen presents and develops at this point the spousal symbology of the spiritual couple,

116 Cf. Mt 7, 6.

117 Origenes, Homilia Prima in Genesin 17, in: Origenis, Opera omnia quae graece vel latine tantum extant et eius nomine circumferuntu, 129: "[...] sensum Christi accipere, et scire «quae a Deo donata sunt nobis»: et quae nobis ad escam data sunt, non faciamus escas porcorum, vel canum [...]” (traduzione italiana: M.I. Danieli (ed.), Origene, Omelie, 61).

118 Cf. Gv 14, 23.

119 Cf. 1 Cor 6, 19.

120 Origenes, Homilia Prima in Genesin 17, in: Origenis, Opera omnia quae graece vel latine tantum extant et eius nomine circumferuntu, 129-130: "[...] quibus dignum sit suscipi in hospitio cordis nostri verbum ac filium Dei venientem cum patre suo, et volentem facere apud nos mansionem in Spiritu sancto, cuius prius templum per sanctitatem debemus existere" (traduzione italiana: M. I. Danieli (ed.), Origene, Omelie, 61-62). 
from whose harmony the dominion over the "earth" springs, meaning the dominion over the sensus carnis and the corporeae voluptates.

Origen observes that "our interior man is made of spirit and soul: the spirit is said to be the male, and the soul can be called the female". He indicates in the concordia and the consensus-with an evident reference to the theme of $\sigma u \mu \phi \omega v i \alpha$ between the spouses (see 1 Cor 7: 5) -the two elements of the necessary condition for the procreation of children, meaning of those good thoughts capable of populating the "earth", identified with the subiectum sibi sensus carnis.

Origen is the first author to introduce such idea and its specific language when widely explaining creation. The "interior human being" is conceived as dychotomic with reference to the corporeal, material human being and as following a totally opposite direction. The "image" - or, rather, the being made at the image ( $\kappa \alpha \tau$ ' $\in i \kappa o ́ v \alpha$ ) - can be affirmed solely of the "interior human being" inasmuch as it identifies with the superior part of the soul ( $\psi \cup \chi \eta$ ), called "intellect" (voû $\varsigma$, mens, intellectus), which is the subject of reason ( $\lambda$ ó $о \varsigma)$.

Origen is quite conscious of the problem of the dualism-in fact, the oppositionbetween the interior and the exterior human being, as found in St. Paul, whose doctrine resonates in Origen's theological stress of the visible and the invisible.

The opposition between the interior and the exterior human being, as well as their analogy, is extended also to the human functions, where two ways of the intellect are

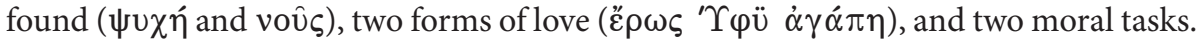

Origen's way of arranging the discourse on the creation of the human being leads him to use the spousal symbology and the feminine character of the soul, considered as inferior with reference to the spiritus, since it is right the responsibility of the soul to push the human being towards corporeal and earthly passions, presented in terms of adultery.

In conclusion, after having recalled-through a literal exegesis-the "dominion" over the animals carried out by the first couple, Origen presents-through a spiritual exegesis-their "dominion" over vices, both springing from the soul and from the body.

\section{Streszczenie}

Orygenes wygłosił wszystkie Homilie na Księgę Rodzaju w Cezarei Palestyńskiej mniej więcej w połowie III wieku. Dysponujemy nimi dzisiaj jedynie dzięki łacińskiemu przekładowi, którego dokonał Rufin z Akwilei w latach 400-404. Homilie te ukazują nam Orygenesa dojrzałego, który jest już prezbiterem. Kluczem do zrozumienia Księgi Rodzaju i całego Pisma świętego, także Starego Testamentu, jest Jezus Chrystus.

W niniejszym artykule analizujemy rozdziały od dwunastego do siedemnastego, czyli do końca Pierwszej Homilii Wielkiego Aleksandryjczyka na Księgę Rodzaju. Jest to bowiem owa część tegoż kazania, w której Orygenes bada stworzenie człowieka.

W omawianej części Homilii Orygenesa spotykamy charakterystyczną dla Aleksandryjczyka egzegezę opartą na wzajemnym zazębianiu się, uzupełnianiu i wypełnia- 
niu znaczenia „historycznego” opisanych wydarzeń ze znaczeniem duchowym, alegorycznym.

Orygenes podkreśla godność i wielkość człowieka, którego bezpośrednio stwarza sam Bóg, w przeciwieństwie do innych, podstawowych rzeczywistości kosmicznych. Człowiek ponadto, szczególnie „człowiek wewnętrzny”, jest “obrazem” Chrystusa-Logosu. Aleksandryjczyk podkreśla to, polemizując z rozpowszechnionymi wówczas poglądami antropomorficznymi Boga.

Człowiek jednak, który został stworzony „na obraz i podobieństwo Boga”, popełniwszy grzech pierworodny, przyjął obraz diabła, który zostaje unicestwiony, gdy człowiek zdobywa podobieństwo Chrystusa.

Stworzenie „mężczyzny i kobiety” Orygenes wyjaśnia dosłownie jako antycypację przyszłego stworzenia kobiety, podczas gdy według znaczenia duchowego stworzenie to oznacza człowieka wewnętrznego ukształtowanego ex spiritu et anima. Orygenes stwierdza, że „nasz człowiek wewnętrzny składa się z ciała i duszy: duch jest mężczyzną, a ciało - kobietą". Grzeszny człowiek musi więc odnaleźć w sobie człowieka wewnętrznego.

Pierwsza Homilia Orygenesa na Księgę Rodzaju kończy się nawiązaniem do Nowego Testamentu: 1 Kor 2, 16, 1 Kor 2, 12. Teksty te, w świetle których należy czytać analizowany przez nas fragment Pisma świętego, powinny uczynić nas "godnymi, aby przyjąć w głębi naszego serca Słowo i Syna Bożego, który przybywa ze swoim Ojcem i chce mieszkać w nas w Duchu Świętym, którego mamy być przede wszystkim świątynią ze względu na świętość". 